\title{
New Technologies to Develop Wireless Networks
}

\author{
Saeed M Alshahrani \\ Department of Computer Science, Sacred Heart University, Bridgeport, CT, USA
}

\begin{abstract}
Smartphones have become a revolution within the last years. The increase of the computing and communication capacity of these devices allows them to perform a wide range of tasks. Newer PDAs (eg, iPhone, Treo, Palm T/X, HP iPAQ) access the Internet to download files, retrieve information, and update software programs directly on the device without having to connect to a desktop or laptop computer. cellular wireless networks location management is an important mechanism that enables the network to discover the current attachment point of a mobile so as to facilitate successful information delivery.
\end{abstract}

Keywords: Wireless, Bluetooth, PDA, GPRS, Networks.

\section{INTRODUCTION}

Regards technology wireless is high-tech whereby used capacity of networks by minimizing the number of locally and internationally. It is alternative for wire, and bottlenecks.

facilitated the process of building infrastructure. It has types and each type has its characteristics and its uses .It has a huge role in our life. Wireless communication wireless communication is enjoying is fasting growth period in history, due to enabling technology which permit widespread deployment.

And some are influenced by circumstances surrounding some of the other don't influenced anything. Today we can not do without it in our daily lives. In the context of communication, there exists a large number of networks which we rely on. For example, telephone networks, Internet, Peer-to-Peer (P2P) networks, wireless ad hoc networks, etc. Significant research has focused on the operations and management of communication networks with the fundamental objective of information transfer in terms of high reliability, security, robustness, and throughput. The Network Coding (NC) (Ahlswede et al., 2000) technique emerged at the beginning of the previous decade with the primary aim of improving the throughput of communication networks. From its inception, the concept of $\mathrm{NC}$ has attracted the interest of researchers who have explored resource utilization improvements and the robustness of various wired and wireless networks.

The NC scheme refines the precise flow of data in a network by transmitting combined digital messages from source to receiver. Prior to NC, the only job of intermediate nodes (i.e., switches and routers) within a network was to forward data packets towards the destination(s). NC ideology encourages that, in addition to forwarding packets, intelligent mixing of packets (from different sources before forwarding) increases the network throughput. Therefore, in network coding, routers and switches have been replaced by coders that allow intermediate nodes to encode the incoming messages and then forward these messages to other node(s). This (encoding and forwarding) scheme increases the effective

In traditional (wired) distributed systems, the group membership problem has been studied thoroughly (we refer to for a survey). The basic premise of group membership is to know which other nodes are there, for instance to share the load of some task. Nowadays wireless LAN or Bluetooth often replace large parts of wired networks since one does not have to build an expensive communication infrastructure first, but can communicate in "ad hoc" mode immediately. This motivates a revisit of the group membership problem in a wireless context: imagine for example a bunch of wireless sensors, distributed in an area to observe that area. From time to time some of the nodes fail, maybe because they run out of energy, maybe because they are maliciously destroyed. On the other hand, from time to time some more sensors are added. Despite this churn (nodes joining and leaving, all nodes should be aware of all present nodes, with small delay only. To account for the selforganizing flavor and the wireless context, we decided to change the name from group membership to selfmonitoring in this paper. We present an efficient algorithm for the self-monitoring problem in an adversarial setting. The relevance of localization for the business model of operators has motivated plenty of techniquesthat are mostly based on a set of available measurements ranging from signal arrival tims to maps of received power. Indeed, current and future $3 \mathrm{GPP}$ radio access networks, through their associated messages in their respective radio resource control (RRC) and nonaccess-stratum (NAS) protocols, provide a set of parameters that allow operators to perform real-time user localization.

\section{BLUETOOTH TECHNOLOGIES}

Information fusion refers to the study of techniques that combine and merge information and data residing at 
disparate sources [1], [2] and [3]. The information fusion is present in many technological areas, including the World Wide Web, where different types of information fusion play an important role. The web influences almost every aspect of businesses and our daily lives [4].

In many senses, Smartphones have become a revolution within the last years. The increase of the computing and communication capacity of these devices allows them to perform a wide range of tasks. Millions of users use their Smartphones daily to perform lots of tasks, such as internet access, global positioning systems, multimedia, smart homes control [5], remote control, and patient monitoring. Among the current mobile platforms we must highlight Android, iOS, Windows Phone and Blackberry, which are the target of millions of mobile applications that are distributed via app stores. Many of these mobile applications merge information from the internet with other information obtained by using the phone's hardware elements, such as Bluetooth, Wi-Fi, sensors, and GPS.

Bluetooth technology which regards transfer data from device to another device it has hold Bluetooth technology, and don't allows access without this tech, Bluetooth network needs Bluetooth access point to connect server with other Bluetooth. ( The Bluetooth protocol stack is split in two parts: a "controller stack" containing the timing critical radio interface, and a "host stack" dealing with high level data). There are lots advantages of Bluetooth we can mention are the following:

- User can get the network in any place in the range of Bluetooth wireless.

- Bluetooth is automatic which means any someone doesn't have to set up a connection or push any buttons.

- Bluetooth technique allow user to send data and voice.

- Bluetooth bandwidth is uses little between pc and phone.

- Bluetooth cost regarding is low in general compared to other tools.

Given the rise of personal networks, mostly by the smartphones, Bluetooth technology is widely disseminated in any form and this makes it a technology that is also commonly found indoors, which has allowed in recent years the exploration of techniques for location based on this technology. To solve the location based on bluetooth you can use the same principle of trilateration getting the RSSI and Link Quality that even when it is not a measure such as the RSS, through some basic operations can be transformed to a distance approximation.

\section{PDA TECHNOLOGY}

Personal Digital Assistant can function as a cellular phone, fax sender, web browser and personal organizer. Most PDA is incorporated handwriting and voices features.

Accept inputs from users and convert these inputs to data and receive signal from anther system and convert these signal to data. There are two main features that put a handheld PDA much above a basic personal organizer. One is its ability to be connected and communicate with many other electronic devices, like a PC or digital camera. The other is the extensive abilities of the PDA software. Together with the third, the "* always with you" feature, all this combines into a powerful and versatile personal assistant." All PDA come with some kind of personal information management software that typically handles some the following task to keep organized like: Track appointment and games, reminder, and takes notes. Some advantages of PDA are:

- A personal digital assistant (PDA) allows you to efficiently accesses , organize, collect, store, and process .

- It is small size you can insert in your pocket and suitable for hand palm.

- It is portable because you can take with you always unlike computer.

- 4Easy to deal user with interface touching

- It is store and retrieve phone number and addresses .

- It can connect to anther electronic device .

- As for PDA software applications such wordprocessing, spreadsheet, games, money management, weight or fitness monitoring, electronic book reading programs, street map PDA software .

- Some PDA allow e-mail and internet access, while on many others you can prepare e-mails .

- Make sure you buy pocket pc, you can read e-books on them.

- It mange your time with easily.

Personal digital assistants (PDAs) have been a part of health care since they came on the market in the 1980s as an organizing device that could be carried easily in a pocket. Today "PDA" represents a large number of hardware devices that range from a simple organizer to interactive wireless devices with two-way communication. These devices include an entire range of smartphones from companies such as Palm, Hewlett-Packard, LG, Nokia, Sony, Research in Motion (RIM), and Apple. Today's PDAs have the ability to download software, store files, record voice, play music and videos, and access the Internet; in short, they have most of the functions of a laptop computer. Unlike desktop or laptop computers they are small enough to fit easily into a pocket and are instanton, instant-access devices.

Newer PDAs (eg, iPhone, Treo, Palm T/X, HP iPAQ) access the Internet to download files, retrieve information, and update software programs directly on the device without having to connect to a desktop or laptop computer. The Blackberry from RIM revised thinking in the marketplace by creating "push e-mail" for the PDA. The most recent handheld device, the Amazon Kindle, is marketed as an electronic reader with a slightly larger form factor than a typical PDA. The Kindle is an informatics warehouse that provides free access to the Internet, downloads e-books, articles, and blogs, searches them, and allows the reader to make notes on them. Newspapers, blogs, and magazines are pushed to the device. The Kindle is the first device to bridge the gap 
between PDA and computer to change the way people access and read materials in a digital world.

\section{IV.GPRS TECHNOLOGY}

General Packet Radio Service is a mobile data service available to users of GSM mobile phones. It is often described as "2.5G". It provides moderate speed data transfer by using unused TDMA channels in the GSM network. First it was standardized by ETSI but now that effort has been 03GPP. the information that was send via GPRS is divided into little packets, so that they can then be transported. By sending information at speeds up to 115 kilobits in this way, the network is used more efficiently and higher speed is achieved. In cellular wireless networks location management is an important mechanism that enables the network to discover the current attachment point of a mobile so as to facilitate successful information delivery.

Location information about mobiles is maintained by registration [1] and [2] and mobiles update their registration area(s) information with the HLR and the VLR, in the event of changes to the registration areas(s) [3]. A registration area comprises of a set of cells and may be static [4] or dynamic [5]. A static registration area is comprised of a set of cells permanently assigned and is fixed for all mobiles, whilst, dynamic registration areas are made to vary in accordance to the traffic conditions of each mobile and its surrounding users so as to achieve optimality of a predefined objective. Although optimality is generally achieved through the use of dynamic registration areas, this scheme imposes a higher computational complexity and incurs more data storage capacity. As a result, most of the existing cellular systems use static registration areas. location updates are to be performed when mobiles cross the partitioning of LA so that up-to-date location information for each mobile is stored in the respective HLR and VLR. It is to be noted that the location information of each mobile is tracked on a resolution that is dependent on the size of the LA.

Hence, to facilitate a successful data delivery, all the cells within the LA have to be paged concurrently. GSM provides only circuit-switched (CS) services, while GPRS provides both packet-switched (PS) services and CS services. In view of its ability to accommodate both bursty packet data services and traditional voice data services, the location management design for GPRS is to be considerably more complex, as compared to GSM. Some advantages of GPRS are New mobile telephones and the newer PDAs are often already GPRS- compatible and it's easy to use a laptop of mobile Internet access via GPRS with a special GPRS card.

Via GPRS, information have much faster access, user can stay always online, much higher transmission speed, can accept phone calls while online, searching internet or WAP contents, Charges on the quantity of the data transferred and access Internet from any point covered with the GSM network signal.

\section{CONCLUSION}

the web applications may be a good alternative to native mobile applications, as they have equivalent communication capabilities and they also have several benefits. Among these benefits is the fact that the web approach eliminates platform dependency and can run on a wider range of mobile platforms with lower development and maintenance costs. Besides, the proposed language does not require the use of specific web technologies, libraries, plugins or other components. This use of distributed intelligence in collaborative communities will infuse goal-oriented innovation into nursing practice. Faculty, preceptors, and students will use existing technology to inspire ideas for better goal-oriented technology - technology that supports real-time evidence based practice.

\section{REFERENCES}

[1] McLeod, R. P., \& Mays, M. Z. (2008). Back to the Future: Personal Digital Assistants in Nursing Education. Nursing Clinics of North America, 43(4), 583-592.

[2] Chai, N., Yeo, B. S., \& Chew, Y. H. (2006). Location management for GPRS. Computer Networks, 50(15), 2888-2901.

[3] Khoor, S., Nieberl, J., Fügedi, K., \& Kail, E. (2001). Telemedicine ECG-telemetry with Bluetooth technology. In Computers in Cardiology 2001 (pp. 585-588). IEEE.

[4] Thompson, B. W. (2005). The transforming effect of handheld computers on nursing practice. Nursing administration quarterly, 29(4), 308-314.

[5] Salkintzis, A. K., Fors, C., \& Pazhyannur, R. (2002). WLAN-GPRS integration for next-generation mobile data networks. Wireless Communications, IEEE, 9(5), 112-124. 